\section{AEET}

ASOCIACIÓN ESPAÑOLA DE ECOLOGÍA TERRESTRE

\section{ecosistemas \\ REVISTA CIENTÍFICA DE ECOLOGÍA Y MEDIO AMBIENTE}

ISSN 1697-2473 / Open access

disponible en www.revistaecosistemas.net

\title{
XV Reunión científica anual de ECOFLOR
} \author{
Santamaría ${ }^{1}$, J. Seguí ${ }^{1}$, A. Traveset ${ }^{1}$. \\ (1) Instituto Mediterráneo de Estudios Avanzados (IMEDEA), UIB-CISC, E-07190 Esporles, Illes Balears, España \\ (2) E.T.S.I.A.A.B. Universidad Politécnica de Madrid, Ciudad Universitaria, E-28040 Madrid, España \\ (3) Universidad Rey Juan Carlos, E-28933 Móstoles, España \\ (4) Estación Biológica de Doñana (EBD-CSIC), E-41092 Sevilla, España \\ *Autor de correspondencia: C. Lara-Romero [carlos.lara.romero@gmail.com]
}

C. Lara-Romero1,*, G. Escribano-Ávila1 ${ }^{1}$, J. Galeano², C. García-Verdugo ${ }^{1}$, J.M. Iriondo ${ }^{3}$, A. Lázaro ${ }^{1}$, F.X. Picó ${ }^{4}$, S

> Recibido el 21 de febrero de 2018 - Aceptado el 21 de febrero de 2018

Lara-Romero, C., Escribano-Ávila, G., Galeano, J., García-Verdugo, C., Iriondo, J.M., Lázaro, A., Picó, X., Santamaría, S., Seguí, J., Traveset, A. 2018. XV Reunión científica anual de ECOFLOR. Ecosistemas 27(1): 132-133. Doi.: 10.7818/ECOS.1564

Los días 1 y 3 de febrero se celebró en Mallorca la XV Reunión anual de ECOFLOR (http://webecoflor.webs.uvigo.es) (Fig. 1), red temática y grupo de trabajo sobre ecología y evolución floral de la Asociación Española de Ecología Terrestre (AEET). Fue organizada por el grupo de Ecología Terrestre del Instituto Mediterráneo de Estudios Avanzados (IMEDEA-CSIC, http://imedea.uib-csic.es/icg/ecol_ terr/cast/group.html).

En la reunión participaron 60 investigadores provenientes de 10 países. Contamos con dos conferenciantes plenarios de gran calidad: Jens Olesen (Aarhus University, Dinamarca) y Juan P. González-Varo (University of Cambridge, Reino Unido), así como 21 charlas de gran nivel científico, casi todas a cargo de estudiantes de doctorado o investigadores posdoctorales. La representación de hombres y mujeres fue equitativa (11:10). Los resúmenes de las charlas se pueden descargar en https://ecoflor2018.weebly.com/program.html.

\section{La red social de ECOFLOR}

Para celebrar los 15 años transcurridos desde la primera reunión, Javier Galeano (Universidad Politécnica de Madrid) presentó la red de afiliaciones de los miembros de ECOFLOR, cuyos enlaces representan co-autoría. La red incluye 87 investigadores que asistieron a ECOFLOR entre 2016 y 2018 y otros participantes asiduos, conectados entre sí a través de unos 150 enlaces (Fig. 2). Es una red social con unas claras características de "mundo pequeño" (sensu Watts y Strogatz 1998, Nature) que además muestra una gran proyección internacional, ya que los 87 integrantes han colaborado a su vez con un total de 3133 científicos de diferentes países. El análisis sólo reconoció a una mujer como investigadora clave dentro del grupo (i.e. con número de publicaciones y de colaboradores significativamente altos). Este resultado demuestra que la brecha de género sigue instalada en el colectivo de ECOFLOR.

\section{Mesas redondas}

Este año se retomó una idea original de ECOFLOR al organizar dos mesas redondas. La primera, con el título "Efectos antropogénicos sobre la evolución floral: hacia una evolución asistida", estuvo

\section{ECOFLOR 2018}

\author{
Caixa Forum Palma, Mallorca \\ February 1st - 3rd 2018
}
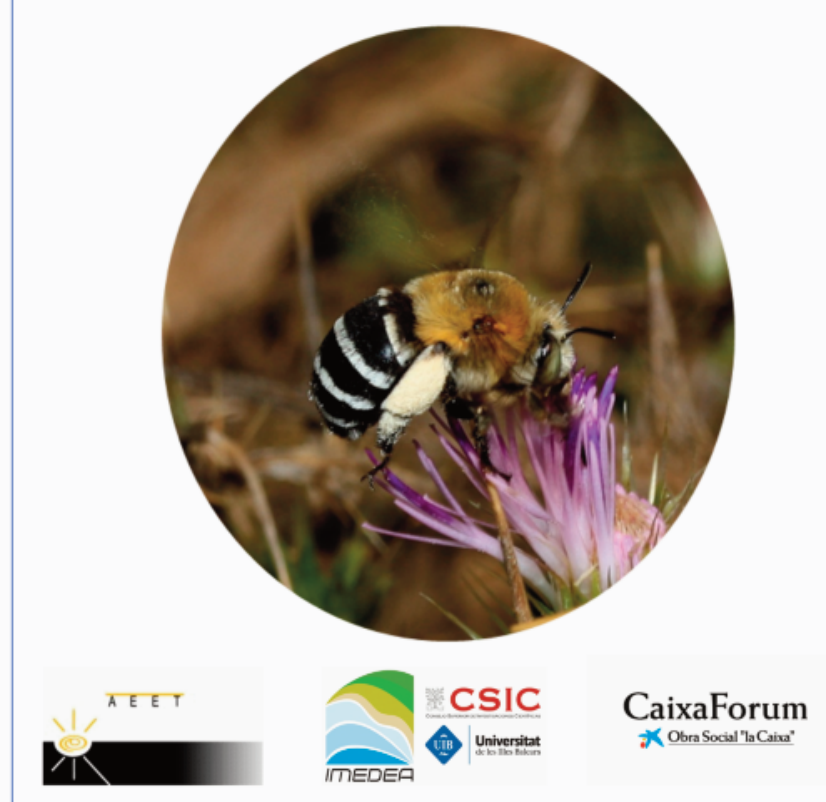

Figura 1. Cartel promocional de la XV Reunión anual de ECOFLOR. 


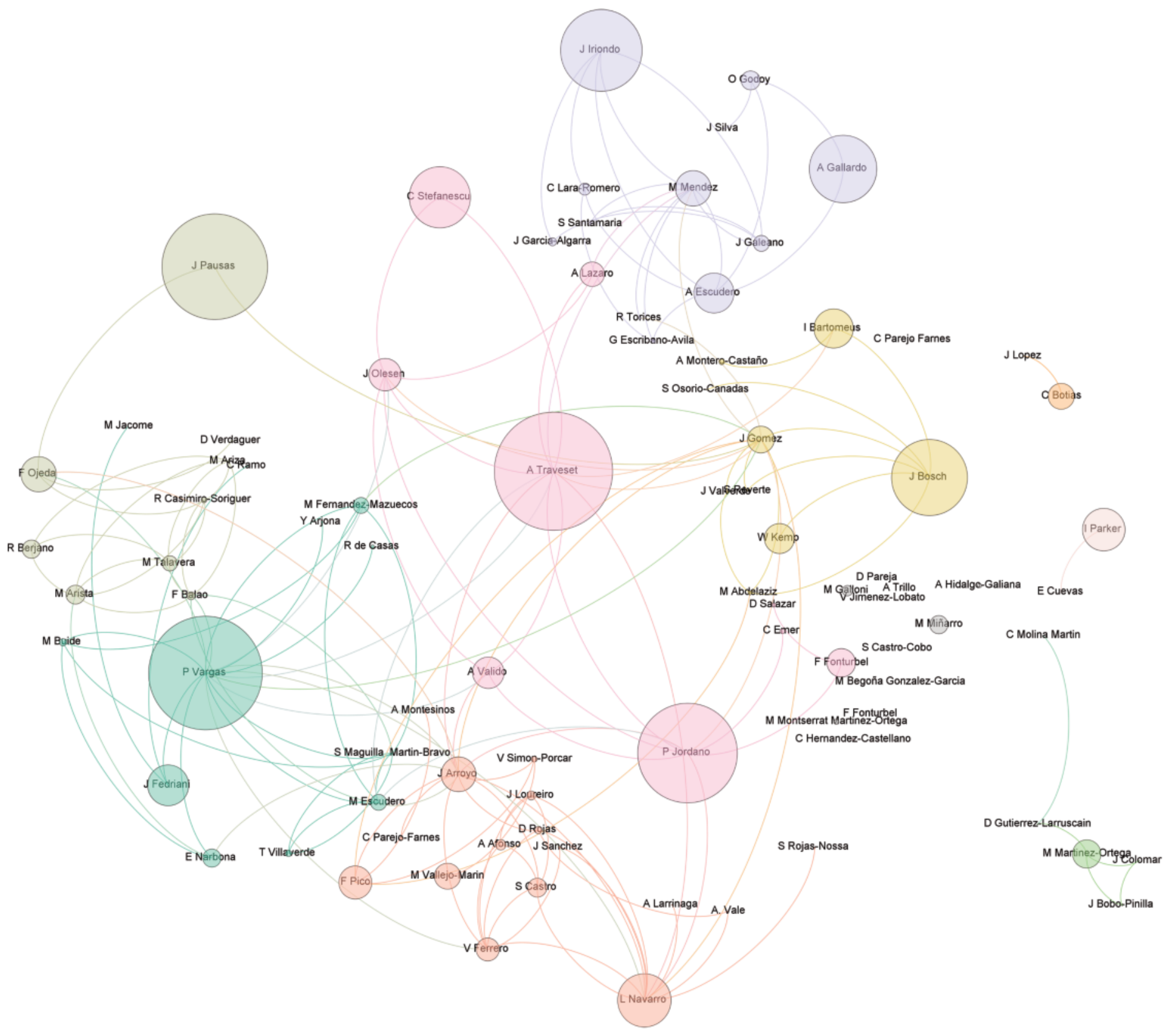

Figura 2. Red de afiliaciones de los miembros de ECOFLOR, cuyos enlaces representan co-autoría. La red incluye 87 investigadores que asistieron a ECOFLOR entre 2016 y 2018 y otros participantes asiduos, conectados entre sí a través de unos 150 enlaces.

moderada por José Iriondo (Universidad Rey Juan Carlos) y contó con la participación de Luis Giménez-Benavides, José M. Gómez, Rocío Pérez-Barrales y Pablo Vargas. La discusión se enmarcó en torno a tres aspectos: 1) ¿Qué tipo de fuerzas y procesos evolutivos resultan afectados por los impactos humanos y cómo afecta esto a las características florales y de los polinizadores?; 2) ¿En qué medida son estos efectos relevantes y en qué sentido?; 3) ¿Deberíamos tener en cuenta estos efectos en las evaluaciones de conservación de la biodiversidad? ¿Tiene sentido intervenir de forma consciente sobre los procesos evolutivos?

La otra mesa redonda, "Ecología evolutiva en Ecoflor: estado actual, principales problemas y retos de futuro", fue moderada por Xavier Picó (EBD-CSIC). Planteó una cuestión fundamental para todas las disciplinas científicas y que muy ocasionalmente puede tratarse de un modo directo y sin tapujos: ¿qué podemos hacer como colectivo para mejorar la ciencia y la ecología evolutiva en particular? Se argumentó la relación que existe entre la situación actual y la escasez de recursos para la investigación debida a la crisis económica. No obstante, quedó clara nuestra fuerza como colectivo para influir sobre lo que sí podemos actuar (e.g. colaboraciones científicas, formación de investigadores) pero también para evitar situaciones estériles que implican un desgaste emocional (e.g. cambiar la política de I+D estatal). La audiencia mostró su satisfacción por haber tenido la oportunidad de debatir y reflexionar sobre aspectos no puramente científicos pero que inciden directamente sobre la actividad científica que realizamos. Finalmente, se insistió en la necesidad de seguir celebrando este tipo de actividades en futuras ediciones de ECOFLOR.

La XVI Reunión de ECOFLOR se celebrará en 2019 y será organizada por los compañeros de la Universidad de Granada. 